\title{
Las políticas de enseñanza de prácticas periodísticas y la producción de periódicos escolares en Argentina y Brasil*
}

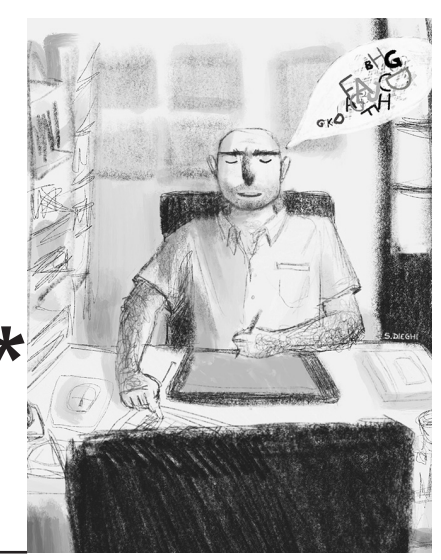

Adair Bonini

Universidad Federal de Santa Catarina - CNPq, Brasil

adair.bonini@gmail.com.

Trabajo recibido el 12 de julio de 2018 y aprobado el 29 de octubre de 2018 .

\begin{abstract}
Resumen
La introducción de prácticas de lenguaje como contenidos curriculares en Argentina y Brasil, a partir de la década de los 90, implica la elección de formas de representar, textualizar y enseñar las mismas. En este artículo se analizan las políticas de enseñanza de prácticas periodísticas en esos dos países, considerándose las políticas elaboradas a nivel nacional por ambos ministerios de educación y a nivel local por los ministerios de educación de las ciudades de Buenos Aires y Florianópolis. En un momento posterior las políticas identificadas son verificadas en un análisis de cuatro diarios escolares, dos de cada ciudad. Todos los materiales analizados comprenden la etapa de la educación primaria en Argentina y de la enseñanza fundamental en Brasil. El trabajo es guiado por las perspectivas teóricas de la Glotopolítica y del Análisis Crítico de Géneros.
\end{abstract}

\section{Policies for the teaching of news practices and the production of school newspapers in Argentina and Brazil}

\begin{abstract}
The introduction of language practices as curricular contents in Argentina and Brazil, beginning in the 1990s, entailed the choice of ways to represent, textualize and teach such practices. In this article, the policies for the teaching of news practices in the two countries are analyzed, both those launched by ministries of education, at the national level, and those launched by education departments of the municipalities of Buenos Aires and Florianópolis, at the local level. After that, the identified policies are checked through the analysis of four school newspapers, two from each
\end{abstract}

* Este artículo reporta una investigación realizada durante una instancia de posdoctorado en la Universidad de Buenos Aires, con la dirección de Elvira Narvaja de Arnoux. Agradezco a la CAPES por la otorga de una beca que resultó fundamental para la realización del postdoctorado (código 88881.121072/2016-01). Agradezco también a Francisco Sánchez por la ayuda en la revisión de lengua española. Asumo total responsabilidad por las opiniones expresadas y por eventuales errores de análisis existentes en el artículo.
Palabras clave

periódico escolar alfabetización mediática enseñanza de lengua materna Glotopolítica géneros discursivos

\section{Keywords}

school newspaper media literacy mother tongue teaching Glotopolitics genre 
city. All the analyzed materials refer to the stage of primary education in Argentina and elementary education in Brazil. The work is guided by the theoretical perspectives of Glotopolitics and Critical Genre Analysis.

\section{Políticas de ensino de práticas jornalísticas e a produção de jornais escolares na Argentina e no Brasil}

\section{Resumo}

A introdução de práticas de linguagem como conteúdos curriculares na Argentina e no Brasil, a partir da década de 90, implica na escolha de formas de representar, discursivizar e ensinar tais práticas. Neste artigo, são analisadas as políticas de ensino de práticas jornalísticas nesses dois países, em nível nacional, em termos de ambos os ministérios da educação e, em nível local, em termos das secretarias de educação das cidades de Buenos Aires e Florianópolis. Em um momento seguinte, as políticas identificadas são verificadas em uma análise de quatro jornais escolares, dois de cada cidade. Todos os materiais analisados compreendem a etapa da educação primária, na Argentina, e do ensino fundamental, no Brasil. O trabalho se orienta pelas perspectivas teóricas da Glotopolítica e da Análise Crítica de Gêneros.

\section{Introducción}

La enseñanza de lenguas a través de prácticas de lenguaje viene ocupando un lugar destacado en las currículas nacionales tanto brasileña como argentina desde la mitad de los años 90 . Inicialmente las prácticas fueron abordadas a partir del concepto de género discursivo y más recientemente también a través del concepto de alfabetización - literaria (Cosson 2006), mediática (Buckingham 2003), digital (Coscarelli y Ribeiro, 2007), etc.

En este trabajo, dentro del conjunto de las prácticas mediáticas de lenguaje, son focalizadas las prácticas periodísticas, en términos de su enseñanza (en especial el trabajo con el periódico escolar) y de las políticas que rigen esa enseñanza. Se consideran los años finales de las etapas de la educación primaria en la Argentina y de la enseñanza fundamental en Brasil, elección que se hace para permitir comparación y también por estar en sintonía con trabajos anteriores del investigador (por ej.: Bonini, 2017).

El artículo busca, en este sentido, responder a la siguiente pregunta: ¿Cómo son las políticas de enseñanza de prácticas periodísticas en Argentina y Brasil y de que forma los periódicos escolares producidos reflejan esas políticas? Para responderla, se examinan los siguientes aspectos: 1) las políticas de enseñanza de prácticas periodísticas a partir de algunas currículas y algunos programas gubernamentales de ambos países y ciudades enfocados; 2) la representación de la práctica periodística movilizada en los periódicos producidos en el interior de esas políticas, focalizando, en el análisis, los siguientes aspectos: la organización del periódico como género, la configuración de los objetos de discurso, la representación de las relaciones sociales y de los roles involucrados.

En consonancia con Fairclough (2003), el término discurso se define en este artículo como "una forma particular de representar alguna parte del

\section{Palavras-chave}

jornal escolar letramento midiático ensino de língua materna glotopolítica gêneros do discurso 
mundo (físico, social, psicológico)” (p. 17). Interesa aquí comprender si las prácticas periodísticas escolares, en contraste con los contenidos curriculares específicos implicados en ellas, colaboran con la construcción de discursos hegemónicos o alternativos a través de las instancias escolares. Tomamos como datos de análisis tanto documentos oficiales del campo educacional cuanto periódicos producidos en escuelas.

\section{Acerca de las políticas de enseñanza de las prácticas de lenguaje}

El debate sobre la enseñanza de lengua materna ha avanzado en muchos países, como por ejemplo Argentina y Brasil, hacia un consenso sobre la necesidad de trabajar los conocimientos lingüísticos de los/las estudiantes a partir de su experiencia efectiva con prácticas de lenguaje (Geraldi 1992; Kaufman y Rodríguez, 1993; Britto 1997; Alvarado 2001). Se va formando también un consenso sobre cuáles son esas prácticas y los lenguajes correspondientes que puedan ser elaborados didácticamente (Halté [1998] 2008); por ejemplo, el periodismo, la publicidad, la legislación, y la comunicación científica.

Se supone que ese contacto de los/las estudiantes con las prácticas de lenguaje durante el aprendizaje escolar debe ser crítico (Freire 1967), lo que implica movilizar en el aula debates en tres ejes: 1) los aspectos sociales y económicos implicados en la producción de estas prácticas; 2) el discurso dominante que la normatiza; y 3) las voces alternativas que, desde otras perspectivas epistemológicas y ideológicas, proponen otras formas de organización de esas prácticas. Este ejercicio de contraposición de voces está presente en la discusión de diversos autores, por ejemplo lo que propone Buckingham (2003), cuando discute la alfabetización mediática, o las reflexiones sobre lectura de Arnoux (2015b) y Voese (2002). En estos términos, un trabajo pedagógico con noticias basado sólo en la concepción de periodismo imparcial y objetivo estaría privilegiando únicamente el discurso dominante y dejando poco campo para la comparación y la reflexión de los/ las estudiantes (Bonini 2017). Lo mismo se puede decir de una enseñanza de la comunicación científica que privilegie sólo el discurso de la ciencia positiva, sin considerar las propuestas que defienden una producción de conocimiento más cualitativa e interpretativa, lo que implica también un discurso diferente para la ciencia.

Hay en la sociedad (en términos de los diversos grupos que la componen) un juego de poder entre las versiones dominantes y no dominantes de las prácticas de lenguaje, lo que se expresa en los instrumentos que se crean para regularlas como, por ejemplo, los manuales de redacción y los diccionarios técnicos (forense, comercial, de publicidad, científica, etc.). Es el caso de los manuales de estilo del periodismo que en general expresan el discurso hegemónico sobre la producción y circulación de noticias. Además, los periódicos dominantes en Argentina y Brasil (los de la prensa burguesa) también buscan sedimentar su discurso a través de programas educativos, por ejemplo los programas del periódico en la escuela (mediante los cuales se ofrecen ediciones y metodologías de trabajo didáctico estratégicamente preparadas) o los programas de visitas guiadas a las redacciones (a través de las cuales se produce un culto al modo de producción dominante del periodismo). En otros términos las prácticas de lenguaje que son objeto de enseñanza y aprendizaje ya están marcadas por políticas de regulación propias de cada esfera social cuando llegan a las currículas y a la escuela. 
La esfera educativa desarrolla políticas de enseñanza a través de una serie de dispositivos como, por ejemplo, las currículas, las leyes, las resoluciones ministeriales, los programas gubernamentales, la gestión de materiales didácticos, y los programas de evaluación de la educación. El hecho de que las prácticas de lenguaje ganen espacio en las políticas educativas de la enseñanza de lengua materna es un aspecto bastante favorable, si se piensa en una escuela que pueda favorecer la emergencia de identidades participativas, democráticas y politizadas. Por otro lado, si no hay espacio para pensar las versiones dominantes y no dominantes de las prácticas, muchas veces se puede estar reproduciendo relaciones de poder altamente desiguales e injustas o usando la escuela como dispositivo de propaganda de la versión dominante de la práctica.

En lo que se refiere al presente artículo, específicamente, está en cuestión la relación entre política de enseñanza de lengua materna y las concepciones de práctica periodística escolar que esas políticas incorporan. Para realizar este análisis, aquí se articulan dos fundamentos teóricos: la Glotopolítica (Arnoux y Valle 2010) y el Análisis Crítico de Géneros (Bonini 2013).

La Glotopolítica estudia acciones específicas (como la proposición de una ley, un manual de uso, un glosario, etc.), casos y ejemplos de intervenciones explícitas sobre la lengua, observando la conexión entre representaciones sociolingüísticas (ideologías lingüísticas, regímenes de normatividad) y relaciones de poder. Más específicamente, la Glotopolítica:

\footnotetext{
[...] estudia las intervenciones en el espacio público del lenguaje y las ideologías lingüísticas que activan y sobre las que inciden. Estas se vinculan con posiciones sociales y la indagación tiende a analizar los modos en que participan en el cuestionamiento, instauración, reproducción o transformación de entidades políticas, relaciones sociales y estructuras de poder tanto en el ámbito local o nacional como regional o planetario. (Arnoux 2015c, 139-140)
}

Las representaciones sociolingüísticas comprenden modos de significar los objetos lingüísticos (lenguas, variedades, hablantes, acentos, registros, géneros, modos de lectura o de escribir, etc.) y, por lo tanto, "evaluaciones sociales de esos objetos y de los sujetos con los que son asociados" (Arnoux y Valle 2010, 3) a medida que constituyen "regímenes de normatividad que, en el seno de [una] colectividad concreta, articulan la relación entre las formas lingüísticas y su valor social" (Arnoux y Valle 2010, 2).

La Glotopolítica estudia las representaciones sociolingüísticas que componen los dispositivos normativos (por ejemplo, manuales de estilo, glosarios, compendios de escritura, retóricas, gramáticas, diccionarios) y prácticas de lenguaje específicas (por ejemplo, ensayos y artículos periódicos, textos literarios, cancioneros, programas de enseñanza de idiomas) (Arnoux 2015a, 9). Esta disciplina enfocará la lengua, el habla o el discurso al investigar la actuación estatal, de colectivos (partidos políticos, medios gráficos, audiovisuales, digitales, etc.) o de personas. Conforme apunta Arnoux (2015a, 9), parafraseando a Marcellesi y Guespin (1986), esa actuación de los agentes sociales ocurre:

...tanto sobre la lengua (cuando una sociedad legisla respecto de los estatutos recíprocos de la lengua oficial y de las lenguas minoritarias) como también sobre el habla (cuando reprime tal o cual uso en una u otra) o sobre el discurso (cuando la escuela decide convertir en objeto de evaluación la producción de un determinado tipo de texto). 
En ese sentido, al estudiar manuales de estilo de periodismo digital, Arnoux (2015c) accede a un buen panorama sobre la representación del lector y de la lectura en el periódico. Según la autora, los manuales describen ese lector como joven, con tendencia al descubrimiento y a la innovación, pero, contradictoriamente, eses manuales también dicen que es un lector que no puede leer textos muy extensos, o complejos, y al cual se le asigna una inteligencia media, de modo que el texto necesita ser facilitado (por ejemplo, usándose un estilo de lenguaje telegráfico) y excitante (por ejemplo, usándose verbos fuertes al principio del párrafo). Se trata de lector para el cual: "No se vislumbra la posibilidad de ningún gesto 'meta' o crítico" (Arnoux 2015c, 18).

En términos de sus proposiciones teóricas, la Glotopolítica tiene muchos puntos de proximidad con el Análisis Crítico de Géneros (ACG), puesto que ambas tienen como objetivo reflexionar sobre el modo como el lenguaje intermedia las prácticas sociales, especialmente teniendo en cuenta que las relaciones de poder en la sociedad son a menudo asimétricas e injustas. Como explica Bonini (2013), el género, como forma relativamente estable del enunciado (Bajtín, [1953]1985), es un elemento que tiene una función central en la realización de las prácticas sociales. En ese sentido, a través de su estudio se puede verificar su rol en el mantenimiento o cambio de relaciones injustas y desiguales.

Asumiendo algunos postulados teóricos de Fairclough (2003), la ACG desarrollada por Bonini (2013) propone una perspectiva de desfamiliarización reflexiva de las representaciones naturalizadas que sustentan prácticas desiguales. ${ }^{1}$ Partiendo de Freire (1967), Bonini propone, además, que el análisis pueda tener como norte los procesos sociales de transitividad de la conciencia. O sea, es un abordaje que toma como eje de reflexión la emancipación humana y la constitución de una sociedad menos desigual, donde haya apertura para los intercambios significativos, donde los sujetos sociales puedan constituir conocimientos sobre su posicionamiento en el contexto y así colocarse críticamente.

En esta propuesta (Bonini 2013), el estudio de los géneros discursivos es un modo del investigador o de la investigadora formar parte de la resolución de problemas sociales. La investigación en esta perspectiva exige que se elija cierto problema (o práctica) social como foco de trabajo y que se establezca la relación entre ese problema y uno o varios géneros discursivos que lo sostienen o lo transforman. De este modo, se puede verificar cómo determinado género es usado para mantener relaciones desiguales, como en el estudio sobre la representación estratégica de la violencia en un programa de la Rede Globo (Ferretti-Soares y Bonini 2017), o verificar la manera como estudiantes de determinada escuela pueden desarrollar una relación crítica con los géneros de un periódico escolar (Bonini 2017) o con un blog periodístico (Rechetnicou, Lima y Bonini 2016).

Para el presente estudio, se hace un relevamiento de las políticas y discursos que sostienen el trabajo con las prácticas del lenguaje periodístico en la escuela en Argentina y Brasil. En ese sentido, son estudiadas las representaciones sociolingüísticas que constituyen las prácticas periodísticas puestas en funcionamiento en las escuelas, principalmente aquellas que están implicadas en la escrita y la lectura de géneros periodísticos. Esas representaciones sociolingüísticas son buscadas en documentos oficiales de la gestión de la educación.
Las reflexiones aquí expuestas sobre ACG en muchos puntos coinciden con las de Motta-Roth y Heberle (2015). 
A partir de ese relevamiento, se observa cómo las representaciones de la práctica periodística presentes en las políticas se relacionan con algunos periódicos escolares (género discursivo) producidos en dos ciudades: Buenos Aires y Florianópolis. En ese caso, son considerados varios aspectos relativos al discurso como una forma de práctica social: sea la forma del discurso (del periódico como género), sean los temas abordados en eses periódicos, sea la imagen que se proyecta de las relaciones sociales y de los sujetos involucrados. Estos son aspectos característicos en el Análisis Crítico del Discurso de Fairclouh (2003), tomados aquí, sin embargo, como elementos del funcionamiento social del género discursivo. El foco está puesto sobre el género como elemento nuclear de la enunciación, como forma relativamente estable del enunciado (Bajtín, [1953]1985).

Hay que considerar, complementariamente, tres otros aspectos todavía. El primero es que los periódicos (diarios o revistas) son aquí vistos como géneros, una vez que contienen las tres características apuntadas por Bajtín para ser género: tema, organización composicional y estilo. Son, en verdad, grandes géneros formados por otros géneros, o hipergéneros según el término de Bonini (2008). En segundo lugar, hay que explicitar que la elección de estas ciudades de Florianópolis y Buenos Aires se debe al hecho de que son los lugares donde están las universidades involucradas en la investigación: Universidad de Buenos Aires y Universidad Federal de Santa Catarina. Se resalta, por último, que el estudio tiene carácter exploratorio, una vez que fueron analizados solamente cuatro periódicos.

Aclárese, además, que la comparación entre Brasil y Argentina se vuelve un tanto compleja, en función de que sus sistemas educacionales son relativamente distintos en términos de las etapas formales. En el caso de Argentina, hay dos sistemas: uno con escuela primaria y secundaria de 6 años cada una; y otro con escuela primaria de 7 años y secundaria de 5 años. El que rige en Buenos Aires es el segundo. En Brasil hay un sistema nacional que comprende la enseñanza fundamental de 9 años y la enseñanza media de 3 años. Nuestro análisis comprende los años finales de la escuela primaria (Argentina / Buenos Aires) y de la "enseñanza fundamental" (Brasil / Florianópolis), cuando los estudiantes ya concluyeron el proceso de alfabetización inicial (conforme el Cuadro 1).

\begin{tabular}{|c|c|c|c|c|c|}
\hline \multicolumn{3}{|c|}{ ARGENTINA / Buenos Aires } & \multicolumn{3}{|c|}{ BRASIL / Florianópolis } \\
\hline Año-Edad & Ciclo & & Año-Edad & Ciclo & \\
\hline $1-6$ & \multirow{3}{*}{1} & \multirow{9}{*}{ Escuela primaria } & $1-6$ & \multirow{3}{*}{1} & \multirow{5}{*}{$\begin{array}{c}\text { Enseñanza } \\
\text { fundamental - años } \\
\text { iniciales }\end{array}$} \\
\hline $2-7$ & & & $2-7$ & & \\
\hline $3-8$ & & & $3-8$ & & \\
\hline $4-9$ & \multirow{2}{*}{2} & & $4-9$ & \multirow{2}{*}{2} & \\
\hline $5-10$ & & & $5-10$ & & \\
\hline $6-11$ & \multirow{4}{*}{3} & & $6-11$ & \multirow{2}{*}{3} & \multirow{4}{*}{$\begin{array}{c}\text { Enseñanza } \\
\text { fundamental - años } \\
\text { finales }\end{array}$} \\
\hline \multirow{3}{*}{$7-12$} & & & $7-12$ & & \\
\hline & & & $8-13$ & \multirow{2}{*}{4} & \\
\hline & & & $9-14$ & & \\
\hline
\end{tabular}

Cuadro 1: Etapas consideradas en la investigación. 


\section{Políticas de enseñanza de prácticas periodísticas en Brasil y Argentina}

Las prácticas de lenguaje periodístico se abordan en los contenidos curriculares tanto en Brasil como en Argentina. En ambos países son desarrolladas por caminos distintos, pero con representaciones sociolingüísticas parecidas en muchos aspectos.

En Brasil, a nivel nacional, las prácticas de lenguaje ganan espacio en las escuelas de enseñanza fundamental con los Parámetros Curriculares Nacionales (Brasil 1998). ${ }^{2}$ En este documento curricular el foco de enseñanza del lenguaje deja de ser la gramática y pasa a ser el texto en su funcionamiento social.

En términos de las acciones desencadenadas después del documento curricular de 1998, no se propuso un programa específico de alfabetización mediática; el tema, sin embargo, aparece contemplado en políticas públicas. En lo que se refiere a la enseñanza fundamental, forma parte de las acciones del programa Mais Educação, que objetiva ampliar la carga horaria para los/las estudiantes, a través de proyectos y actividades desarrolladas en el contraturno. Entre varios campos de actuación (cinco en 2013), ${ }^{3}$ hay un campo "Comunicación, uso de medios y cultura digital y tecnológica", con diversas posibilidades de trabajo, que contempla incluso experiencias de producción de periódico escolar, siendo este presentado como un
2. En febrero de 2018 el Ministerio de Educación homologó un nuevo documento curricular, cuya tercera versión (Brasil, 2017), producida tras el derrocamiento de Dilma Rousseff, apaga lo esencial de las dos versiones anteriores que fueron ampliamente debatidas con la sociedad.

\begin{abstract}
(1) Exercício da inteligência comunicativa compartilhada com outras escolas e comunidades objetivando a promoção de uma cultura de respeito aos direitos e liberdades fundamentais, da prática democrática e solidária por meio de atividades que valorizem o respeito às diferenças, valorize a diversidade étnico-racial, cultural, geracional, territorial, corporal, de gênero e diversidade sexual, de nacionalidade. Construção de propostas de cidadania engajando os estudantes em experiências de aprendizagens significativas. (Brasil 2013, 13 - cursiva añadida)
\end{abstract}

En ese texto orientador del programa se observa una representación de los medios como fórum público de debates, y del aprendizaje de prácticas mediáticas como un desarrollo de la actuación ciudadana, de hecho que pone el concepto de ciudadanía a servicio de los medios de comunicación. Así, el documento presenta una imagen favorable e idealizada de los medios de comunicación de masa, representación donde las relaciones de poder y los conflictos de intereses no se cuestionan. Al no especificar los tipos de medios de comunicación (comercial, comunitario, gubernamental, institucional, etc.) y sus propuestas y disputas ideológicas, el programa favorece una lectura errada de los medios dominantes comerciales como dispositivos de ciudadanía, democracia y pluralidad. La cuestión es que, por su papel de portavoz del gran empresariado local y extranjero, no han sido pocas las oportunidades en las cuales el periodismo de las corporaciones de comunicación brasileñas y argentinas han desarrollado una práctica exactamente opuesta a lo que anuncian en sus discursos de auto identidad. Tienden así a incentivar al odio entre grupos (el odio a la diversidad, por ejemplo), a linchar públicamente ciertos atores sociales y a no respetar a los derechos y libertades fundamentales.

Un poco antes, en 2011, el Ministerio de Educación brasileño lanzó la Guía de Tecnologías Educativas, que pretendía ser una herramienta de auxilio a las escuelas en la "decisão sobre a aquisição de materiais e tecnologias
3. El programa fue instituido por el Acto Normativo Interministerial $\mathrm{n}^{\circ} \mathbf{1 7}$, de 24 de abril de 2007 , con un consejo operacional que evaluaba y realizaba ajustes año a año. No hay documentos disponibles de todos los años. 
para uso nas escolas brasileiras de Educação Básica pública” (Brasil 2011, 13). En esta guía consta la posibilidad de adquisición de un asesoramiento en la producción de diarios escolares, ofrecido por la ONG Comunicação e Cultura. El trabajo con prácticas periodísticas es representado como una formación para la ciudadanía vía medios únicamente por la justificativa de que él/la estudiante va ocupar el lugar simbólico de un periodista (y non por el ejercicio de lectura crítica de textos periodísticos e del modo como son producidos). El documento afirma que el proyecto de periodismo escolar:

(2) Tem por objetivo fornecer às escolas o jornal escolar como uma ferramenta de uso transversal e interdisciplinar, com base nos pressupostos de que: o jornal é um portador de texto; [realizá-lo é uma forma de educar para a cidadania, ao conectar o aluno de outra forma, no lugar de redator, com a realidade local; fornecer material, através da utilização dos textos do jornal, para o ensino contextualizado.]. (Brasil 2011, 24 - cursiva añadida)

En el material didáctico producido por esa ONG, la Guía del periódico escolar (Comunicação e Cultura 2010), la exposición sobre el periódico escolar también se muestra bastante marcada por el discurso de los medios dominantes:

(3) O Jornal Escolar é pluralista; ele não pratica censura nem oculta informações;

O Jornal Escolar tem finalidade social; ele não faz promoção pessoal ou partidária;

(Comunicação e Cultura 2010, 5 - cursiva añadida)

Cuando se dice que el periódico que producirán los/las estudiantes deberá ser pluralista y neutro (sin voz partidaria), se supone que ese es o debería ser el patrón de acción del periodismo dominante. Se reproducen así los dogmas que circulan en manuales de estilo de las empresas periodísticas, y se establece aquí también una relación directa con la formación para la ciudadanía:

(4) Escrever no jornal permite que a criança construa a consciência de si, em sua relação com o mundo. "Eu faço chegar minha mensagem 'às pessoas". A criança que escreve no jornal está se manifestando como cidadã, e uma escola habilidosa saberá aproveitar o momento para trabalhar o surgimento de um imaginário positivo sobre a participação social. (Comunicação e Cultura 2010, 9 - cursiva añadida)

A la ciudadanía se le presenta, sin embargo, como un discurso técnico, con un valor de verdad que está por encima de las ideologías, de los valores y de las decisiones políticas, siendo expuesta como un "imaginario positivo". La formación crítica también aparece debilitada, como parte de ese discurso técnico, puesto que se la describe como capacidad y non como posibilidad de participación social:

(5) Utilizando o texto livre e o jornal, habituamos os nossos alunos a uma crítica da imprensa, a aceitação e procura dessa crítica. [...] Aprendem, por experiência, a julgar as obras que lhe são apresentadas e rapidamente se tornam aptos a descobrir o que se esconde de falso e contraditório nas imponentes rubricas dos jornais. (Comunicação e Cultura 2010, 16 - cursiva añadida)

En el nivel local brasileño (en términos de nuestro análisis, la ciudad de Florianópolis), no hay programas o acciones específicas relacionadas a la alfabetización mediática. El tema, sin embargo, aparece tratado en los dos documentos curriculares desarrollados por el Municipio (Florianópolis 2008 y 2016). Aquí se hace evidente una representación de los medios de comunicación como actores sociales y, por lo tanto, de sus prácticas como acciones políticas e ideológicas: 
(6)

a) A imprensa é composta por uma série de empresas privadas que visam ao lucro.

b) A maior parte dos ganhos de um jornal advém da publicidade; quanto maior o número de leitores (aumentando a tiragem), mais caro será o espaço publicitário, o que estabelece a competição entre as empresas concorrentes.

c) A notícia pode ser vista, ideológica e politicamente, como um capital simbólico: 0 poder de informar cria relações complexas com o poder econômico e com o poder político.

(Florianópolis 2008, 77 - cursiva añadida)

En ese texto aparece la cuestión del periodismo como empresa, como agente político y de su relación con la publicidad. En el documento más reciente, se explicita un discurso casi opuesto al que explicita el MEC-Brasil (con su pedagogía de la ciudadanía a través de los medios), enfatizando aquí la necesidad de reflexión crítica en lo que se refiere a la producción de medios escolares.

(7) [...] importa [à escola] o cuidado para não se mimetizar com usos das tecnologias que têm lugar fora dela, muitas vezes rendendo-se a pressões de aceleração dos tempos e volatilização dos espaços, como tende a acontecer nas mídias eletrônicas em interações sociais diversas. (Florianópolis 2016 - cursiva añadida)

En la Argentina, al igual que en Brasil, la currícula de los años 90 introduce la enseñanza de prácticas de lenguaje (Argentina 1995). Impresiona la similitud conceptual entre ambas, siendo que la currícula argentina presenta la misma dinámica de práctica y reflexión sobre el lenguaje. Entre los contenidos aparecen con realce las prácticas de producción y lectura de textos periodísticos. El tema es retomado y reformulado en el documento posterior (y importantísimo a esta análisis) de los Núcleos de Aprendizajes Prioritarios (Argentina 2005 y 2011) en el conjunto de los ajustes que son englobados por la Ley de Educación Nacional (Argentina 2006a).

Entre las políticas del MEC-Argentina, fue propuesto un programa relacionado a la alfabetización mediática: Escuela y Medios, que produjo un conjunto relevante de materiales, y que incluía una guía práctica y efectiva de orientación a profesores (Argentina 2006b) sobre la producción de medios en la escuela, incluso el periódico escolar. A pesar de la evidente organización y productividad del programa, su discurso reproduce el discurso de los medios periodísticos dominantes, como por ejemplo cuando expone la idea que todos los periódicos son diferentes, cuando en realidad la mayoría es ideológicamente similar, lo que es fruto de una actuación política de grupo, en conjunto, de las empresas de comunicación,; reproduce ese discurso dominante también cuando plantea la idea de que la consulta de los medios dominantes es una necesidad básica en la actual sociedad:

(8) Existen medios de comunicación locales, provinciales, nacionales y extranjeros. Cada uno difunde hechos que ocurren en distintos barrios, pueblos, ciudades o países. Lo más importante es que cada uno de ellos brinda distintas perspectivas sobre los problemas. Permiten saber qué cosas ocurren cerca y lejos del lugar donde vivimos. (Argentina 2006b, 42 - cursiva añadida)

Esta representación hegemónica de la práctica periodística aparece también cuando la guía explica la clasificación y jerarquización de los hechos publicados como resultado de la importancia de los hechos y no de su función política en el desencadenamiento de nuevos hechos. Aparece también 
cuando la guía afirma que los titulares son escritos de determinada forma para llamar la atención del lector, sin mencionar el papel político de los elementos lingüísticos que se elige para componer estos titulares:

(9) Durante una semana registren hechos que ocurran en la escuela, el barrio y la ciudad en la que viven. Una vez recolectada la información debatan cuáles son los hechos más importantes y seleccionen 506 de ellos. Armen la tapa de un semanario barrial mural. Escriban títulos que llamen la atención del lector y decidan si los acompañarán con imágenes. (Argentina 2006b, 43-44 - cursiva añadida)

Esta emulación de la representación lingüística dominante de la práctica periodística es sintetizada en este pasaje, en el cual la noticia es presentada como un hecho verdadero, reciente y de interés público:

(10) Como primera medida, la noticia debe presentar un hecho verdadero. Además, su contenido debe haberse hecho público recientemente, y tiene que interesarle a mucha gente, es decir debe ser relevante socialmente. (Argentina 2006b, 46 - cursiva añadida)

En general, las políticas del MEC-Argentina también describen el trabajo con periodismo escolar como una formación para la ciudadanía, como evidencia la presencia constante de la palabra ciudadanía en el material o cuando se afirma que la información es "uno de los más preciados derechos cívicos" (Argentina 2006b, 36). Es lo que muestra, además, un artículo de la coordinadora del programa (Morduchowicz 2009):

(11) La investigación sobre las instituciones de los medios contribuirá a construir un conocimiento más profundo acerca de quienes producen mensajes. Los alumnos aprenden acerca de cuestiones relacionadas con la libertad de prensa y la libertad de expresión, el papel del Estado como regulador de estas libertades, la censura y la autocensura, la función social de los medios en democracia y en contextos autoritarios, la propiedad de los medios y el significado de los multimedios. (2009, 16 - cursiva añadida)

La discusión sobre libertad de imprenta se apoya en el Estado como dispositivo regulador, no haciendo mención al poder de las empresas sobre la misma. Esta representación de la ciudadanía como consecuencia del rol mediador del periodismo también está presente en el documento de la UNESCO, en el cual Morduchowicz tiene participación, donde los medios aparecen como "herramientas para la libertad de expresión, el pluralismo, el diálogo intercultural y la tolerancia [...]” (Unesco 2011, 25).

El trabajo de esa autora no se relaciona centralmente a la producción de medios escolares, sino al uso del periódico como material escolar; y de ahí se observa una propuesta más orientada a la cogestión de la educación sobre los medios con las empresas del área (Morduchowicz 2001). Así la formación crítica sostiene un sentido bien más técnico, de hecho que las actividades propuestas (Morduchowicz, Marcón y Minzi 2004) son en general de reconocimiento de rasgos, hacía una alfabetización autónoma (Street, 1985). ${ }^{4}$

En los últimos años, aparece el Programa Conectar Igualdad, que va a desarrollar una línea de trabajo similar al de Escuela y Medios, de alineación al periodístico hegemónico y de trabajo cooperativo con las empresas del área, como se verifica en Albarello (2011). ${ }^{5}$

En el nivel local, aunque la currícula no tenga un abordaje diferente (Buenos Aires 2004), hay un programa de alfabetización mediática: "Medios en la

4. Sin embargo, no se trata de tomar el trabajo de Morduchowicz como errado. Al contrario, hay que reconocer que esa rica experiencia del programa Escuela y Medios es esencial para la proposición de alternativas para el trabajo con alfabetización periodística en la escuela. Lo mismo se puede decir de la inconmensurable importancia de las acciones de la ONG Comunicação e Cultura en Brasil y, además, de todas las experiencias de producción de diarios a través del mondo. Es exactamente el resultado concreto de esas iniciativas que fornece el necesario alimento para la reflexión y el planeamiento de nuevas acciones en este terreno.

5. Hubo, además, un gran programa de producción de periódicos digitales en cooperación con la Fundación Telefónica, que se llamaba EducaRed. Aún queda alguna información sobre los periódicos producidos en este proyecto y que se pueden encontrar en: http://eduteka.icesi.edu.co/ articulos/PeriodicoEscolarEjemplos. Consulta en 28 de feb. 2018. 
Escuela", que muestra una representación de los medios de comunicación como actores sociales, y que propone una práctica escolar reflexiva al hablar de "deconstrucción" y de "consumo mediático":

(12) Medios en la Escuela propone articular los medios en la educación con el objetivo de generar oportunidades genuinas de deconstrucción de los saberes adquiridos en el consumo mediático espontáneo. (Buenos Aires, s.f. - cursiva añadida)

Esta propuesta de análisis crítico de los medios también aparece en los talleres puestos a disposición de los profesionales de la escuela, por ejemplo en la expresión "condiciones de producción de medios":

(13) Talleres de iniciación para profesores:

Más que mil palabras: producción de video documental, de ficción o de animación Hacete oír: producción de un programa radial con formato de "revista"

Temati.com: producción de un boletín de investigación en el soporte papel o virtual Con ojos críticos: lectura y análisis de las condiciones de producción de medios.

(Buenos Aires, s.f.)

En términos generales, lo que se verifica en estas políticas nacionales, tanto en Brasil como en Argentina, es un tratamiento más de promoción que de análisis del periodismo dominante, con reproducción de la versión empresarial de las prácticas periodísticas y su favorecimiento con la asociación directa con el concepto de ciudadanía. En el nivel local, tanto en Florianópolis como en Buenos Aires se enfocan aspectos de la constitución social del periodismo (sus condiciones de existencia) y por tanto ahí se puede verificar una representación más crítica de los medios de comunicación, aunque incipiente.

Hoy se puede ver algo de equivocado en la representación del periodismo como un fórum plural e imparcial de debates democráticos, principalmente se consideramos el rol desempeñado por las grandes empresas de periodismo en la persecución política ocurrida desde el inicio del ciclo de gobiernos progresistas en América del Sur. Sin embargo, cabe mencionar que no se trata de apuntar esas políticas de alfabetización mediática como erro o mala fe, sea por que fueron coherentes, en alguna medida, en el período pos-dictadura civil-militar de nuestros países, sea por que fueron intentos legítimos e importantes de crear políticas en ese campo, de las cuales nos beneficiamos actualmente para nuestra discusión.

\section{Periódicos escolares en Buenos Aires y Florianópolis}

Para el análisis aquí producido, que no es exhaustivo, fueron seleccionados cuatro periódicos (ver Figura 1), dos de Buenos Aires descargados del internet y dos de Florianópolis retirados directamente en las escuelas, los cuales son:

"Somos Argentinos - Escuela N 12 “España” № 12 del D.E. 15, Barrio de Saavedra, Ciudad Autónoma de Buenos Aires, publicado en 2011 (no 4);

" Brisas - Aires de Información - Escuela "Gran Mariscal del Peru Ramon Castilla" №: 12 D.E. 9, Barrio de Colegiales, Ciudad Autónoma de Buenos Aires, publicado en 2009 (año 10, no 11);

》 Comunicação em Outras Palavras - Escola Básica Municipal Vitor Miguel de Souza, Barrio Itacorubi, Florianópolis, publicado en 2011 ( $\mathrm{n}$ o 1); 
" A Voz da José Amaro - Escola Básica Municipal José Amaro Cordeiro, Barrio Morro das Pedras, Florianópolis, publicado en 2010 (año 11, no 25).

Estos periódicos fueron producidos en la misma época, en circunstancias socio-políticas e históricas semejantes, correspondiendo a nivel federal a los gobiernos progresistas de Cristina Kirchner (Argentina), Luiz Inácio Lula da Silva y Dilma Rousseff (Brasil) y en las ciudades involucradas a los gobiernos conservadores/neoliberales de Mauricio Macri en Buenos Aires y Dário Berger en Florianópolis.

Las cuatro escuelas están ubicadas en barrios de clase media, y la población escolar que tienen a cargo pertenece a estratos socio-económicos medios y medio-bajos.

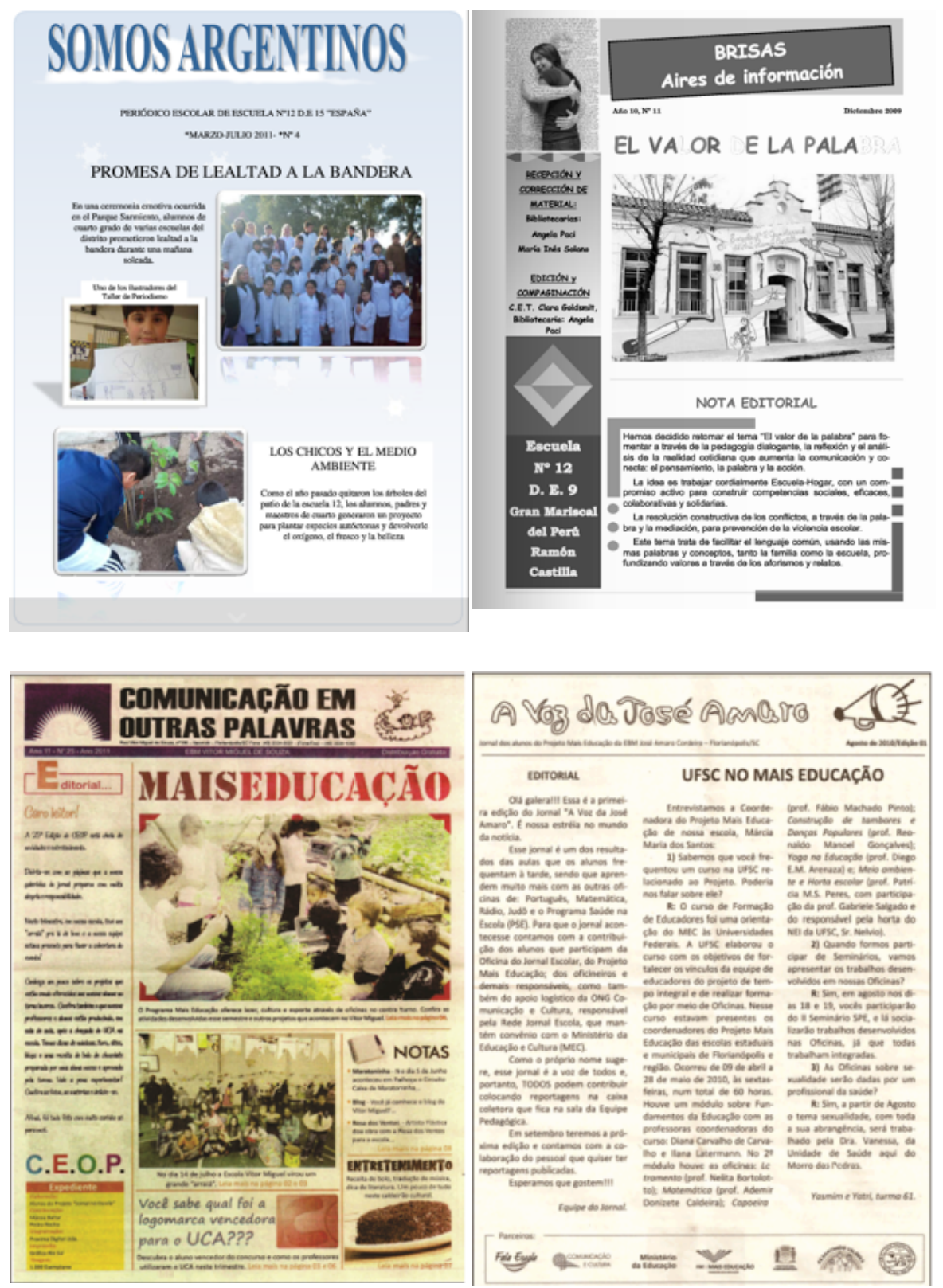

Figura 1: Periódicos analizados. 
Todos los periódicos se relacionan con las políticas educativas analizadas en párrafos precedentes: los dos periódicos de Florianópolis son financiados por el programa Mais Educação, siendo que A Voz da José Amaro explicita textualmente la participación de profesores en talleres del Programa Mais Educação, de la ONG Comunicação e Cultura; los dos periódicos de Buenos Aires también están íntimamente relacionados a los talleres dictados en el Programa Medios en la Escuela.

\section{1. organización}

En lo que se refiere a la organización, los cuatro periódicos tienen vínculos cercanos con los programas educativos que los orientan. Los dos de Buenos Aires reflejan los ejemplos disponibles en el sitio web del Programa Medios en la Escuela (Buenos Aires, s.f.), mientras que, en los de Florianópolis, A Voz da José Amaro presenta el formato indicado por la ONG Comunicação e Cultura y el otro tiene un formato propio.

Los dos periódicos argentinos se muestran como dispositivos de circulación de las informaciones producidas en los talleres y proyectos ofrecidos por la Escuela a los/las estudiantes; en ellos se publican textos como resultado de las actividades pedagógicas. Este aspecto se evidencia en el cuadro 2, especialmente en el periódico Somos Argentinos, cuando los nombres de las secciones hacen referencia a talleres y proyectos pedagógicos; la organización de estos periódicos tiene un tono formal.

\begin{tabular}{|l|l|}
\hline SOMOS ARGENTINOS & BRISAS - AIRES DE INFORMACIÓN \\
- Tapa - encabezado, copetes, fotos & - Tapa - encabezado, editorial, foto, créditos \\
- Créditos & - Agradecimientos \\
- Editorial & - (Presentación o prefacio) \\
- Taller de periodismo & - (textos diversos, los cuales resultan de talleres \\
- Taller literario & y proyectos) \\
- Proyectos naturales & - Indice \\
- Proyecto de ciencias sociales & \\
- Sección divertida & \\
\hline COMUNICAÇÃO EM OUTRAS PALAVRAS & A VOZ DA JOSÉ AMARO \\
- Tapa - encabezado, editorial, copetes, fotos, & - Tapa - encabezado, editorial, texto \\
créditos & - (textos diversos) \\
- (textos diversos) & - Hora del chiste \\
- Música & - Mensajes \\
- Mix cultural & - créditos \\
- Culinaria & \\
- Sueltos & \\
\hline
\end{tabular}

Cuadro 2: Secciones de los períodos analizados.

Los dos periódicos brasileños guardan aparentemente mayor relación con preferencias de la edad de los/las estudiantes, como la sección Música u Hora del Chiste en el cuadro 1; aunque esto no implique que sea un periódico del cual los/las estudiantes se apropien autoralmente.

Los cuatro periódicos ciertamente cumplen funciones pedagógicas relevantes y favorecen aprendizajes específicos que, de otro modo, serían imposibles. Podrían, sin embargo, operar más con la creación y reflexión de los/ las estudiantes, lo que se evidenciaría en las ediciones tanto en términos de la organización de las secciones cuanto de la proposición de temas. Este énfasis en la instrumentalidad pedagógica más que en la actuación de los/las estudiantes, tal vez esté relacionada a la política de uso del periódico escolar a partir da representación dominante del periodismo y, además, basada en 
una práctica ciudadana supuestamente neutra y imparcial (técnica); donde la actuación política y el posicionamiento social de los/las estudiantes se quedan en segundo plano. Para que se privilegie este último aspecto, es necesario profundizar el debate sobre periodismo en aula y producir periódicos a partir de los problemas vivenciados por los/las estudiantes.

\subsection{Objetos de discurso}

Los discursos que conforman los periódicos de Florianópolis y Buenos Aires también se presentan de forma distinta (ver cuadro 3).

Esto ya se evidencia en los nombres de los periódicos, con referencias más patrióticas relacionadas al ejercicio escolar, en el caso de los porteños, y referencias más comunicacionales, ligadas a la representación del periodismo a partir de los medios hegemónicos, en el caso de los periódicos de Florianopolitanos.

\begin{tabular}{|c|c|c|c|c|}
\hline & Somos Argentinos & $\begin{array}{l}\text { Brisas - Aires de } \\
\text { Información }\end{array}$ & Comunicação em Outras Palavras & A Voz do José Amaro \\
\hline $\begin{array}{l}\text { Hechos en la } \\
\text { escuela }\end{array}$ & $\begin{array}{l}\text { Reportes: de } \\
\text { acciones del taller } \\
\text { de periodismo, } \\
\text { plantación de árboles, } \\
\text { del taller de literatura, } \\
\text { del proyecto de } \\
\text { ciencias naturales, } \\
\text { proyecto ciencias } \\
\text { sociales, entrevista } \\
\text { con la directora y la } \\
\text { secretaría }\end{array}$ & $\begin{array}{l}\text { Profesora que se } \\
\text { jubila, presentación } \\
\text { de actividad de } \\
\text { danza, Proyecto de } \\
\text { Mediación escolar, } \\
\text { taller de educación } \\
\text { vial }\end{array}$ & $\begin{array}{l}\text { Noticias: Fiesta de junio, taller de } \\
\text { percusión, taller de capoeira, taller de } \\
\text { alfabetización, taller de producción } \\
\text { del periódico, taller de huerta, taller } \\
\text { de danza, la computadora escolar } \\
\text {, maratón escolar, donación a la } \\
\text { escuela, blog de la escuela, sala de } \\
\text { medios, elección escolar, encuentro de } \\
\text { formación de profesores, policía en la } \\
\text { escuela, censo escolar }\end{array}$ & $\begin{array}{l}\text { Entrevista con } \\
\text { profesora, } \\
\text { participación de la } \\
\text { escuela en evento, } \\
\text { competencia de judo }\end{array}$ \\
\hline Temas juveniles & & $\begin{array}{l}\text { Proyecto bullying, } \\
\text { Salud, internet, } \\
\text { seguridad en la } \\
\text { internet }\end{array}$ & & $\begin{array}{l}\text { Salud, crack, abuso } \\
\text { sexual, infecciones de } \\
\text { transmisión sexual }\end{array}$ \\
\hline $\begin{array}{l}\text { Atividades extra } \\
\text { escolares }\end{array}$ & $\begin{array}{l}\text { Entrevista con vecinos } \\
\text { de la escuela }\end{array}$ & & & $\begin{array}{l}\text { época de pesca } \\
\text { de peces locales, } \\
\text { desaparición de la } \\
\text { playa. }\end{array}$ \\
\hline $\begin{array}{l}\text { Textos literarios } \\
\text { y otras } \\
\text { producciones }\end{array}$ & Biografías, cuentos & $\begin{array}{l}\text { Coplas, cuentos, } \\
\text { carta, dibujos }\end{array}$ & & \\
\hline Campaña & & Recoleccion de pilas & & \\
\hline Enciclopédicos & $\begin{array}{l}\text { Descripción de } \\
\text { plantas, descripción } \\
\text { de costumbres } \\
\text { argentinas, fechas } \\
\text { nacionales, día del } \\
\text { himno nacional, } \\
\text { cronología de Manuel } \\
\text { Belgrano }\end{array}$ & $\begin{array}{l}\text { La palabra, } \\
\text { Día del patrono de } \\
\text { la escuela }\end{array}$ & geomática & \\
\hline Instrucción & & Juego & receta culinaria & \\
\hline Testimoniales & & Mi vida en Japón & & \\
\hline $\begin{array}{l}\text { Acciones de la } \\
\text { escuela }\end{array}$ & & $\begin{array}{l}\text { Carta de despedida, } \\
\text { lista de estudiantes } \\
\text { egresados }\end{array}$ & & \\
\hline Entretenimiento & Adivinanzas & & $\begin{array}{l}\text { Letras de música, resúmenes de libros, } \\
\text { biografías. }\end{array}$ & $\begin{array}{l}\text { Sopa de letras, humor, } \\
\text { mensajes. }\end{array}$ \\
\hline
\end{tabular}

Cuadro 3: Temas abordados en los periódicos. 
En este caso específico, sea en Argentina sea en Brasil, se desarrollan temas escolares, como las acciones de cursos y proyectos, y temas propios de la adolescencia más habituales en las escuelas, como el bullying. Sin embargo, el discurso producido se diferencia, puesto que los hechos, en los periódicos brasileños son narrados como aventuras, discurso típico del entretenimiento periodístico, mientras que en el caso de los argentinos son narrados como relatos académicos, en un discurso típico de la producción escolar escrita.

Las secciones de entretenimiento tienden a aparecer en menor medida en los periódicos argentinos, como prueba de una publicación menos guiada por el discurso periodístico hegemónico.

\subsection{Representación de las relaciones sociales}

Como hemos percibido hasta aquí, los periódicos escolares en ambas ciudades constituyen representaciones, modelos, discursos y prácticas diferentes; así también las relaciones sociales se presentan de modo diferente.

En el caso de los argentinos, el tono es más formal y las relaciones más distantes. Hay una relación de aspecto más profesional, donde los participantes son pares y parte de un debate. Es lo que se verifica, por ejemplo, en estos fragmentos de las tapas:

\section{(14) NOTA EDITORIAL}

Hemos decidido retornar el tema "el valor de la palabra" para fomentar a través de la pedagogía dialogante, la reflexión y el análisis de la realidad cotidiana que aumenta la comunicación y conecta: el pensamiento, la palabra y la acción. (Brisas)

\section{(15) LOS CHICOS Y EL MEDIO AMBIENTE}

Como el año pasado quitaron los árboles del patio de la escuela 12, los alumnos, padres y maestros de cuarto generaron un proyecto para plantar especies autóctonas y devolverle el oxígeno, el fresco y la belleza. (Somos Argentinos)

La elección léxica es propia del discurso escolar y profesional ("pedagogía dialogante", "realidad cotidiana", "especies autóctonas"); además queda claro que la voz que se enuncia en primer plano no es la de los/las alumnos/ as, sino la de los/las profesionales de la escuela.

En los periódicos brasileños, la relación más evidente es entre uno que entretiene y otro que es entretenido, lo que queda reflejado en estos fragmentos de las tapas:

\section{(16) EDITORIAL}

Olá galera!!! Essa é a primeira edição do Jornal “A voz da José Amaro”. É nossa estréia no mundo da notícia. (A voz da José Amaro)

(17) Caro leitor!

A $25^{\mathrm{a}}$ edição do CEOP está cheia de novidades e entretenimento.

Divirta-se com as páginas que a nossa galerinha do jornal preparou com muita alegria e responsabilidade. (Comunicação em Outras Palavras)

Se muestra allí una emoción marcada discusivamente, sea por los signos de exclamación, sea por las elecciones lexicales (como por ejemplo "galera", jerga joven e informal para referirse a un grupo de personas, 
que se vuelve aún más informal con el diminutivo, en "galerinha"), o por las expresiones de afecto ("com muita alegria") y de exacerbación ("no mundo da notícia").

Además de lo enunciado, las relaciones también se dan entre informante y consumidor de información, lo que se verifica por el número de menciones a la acción de producir noticia ("é a primeira edição", "a 25a edição", "com as páginas que a nossa galerinha do jornal preparou").

Este énfasis en la diversión, en el lenguaje informal y en la afectividad también revela un efecto de complicidad simulada entre lector/a y productor/a del periódico. Es una imagen proyectada sobre el/la interlocutor/a. Al mismo tiempo, sugiere la conducción de los/las docentes sobre el terreno de la autoría del periódico, lo que se evidencia en este trecho que tiene cierto tono promocional:

(18) Esse jornal é mais um dos resultados das aulas que os alunos frequentaram à tarde, sendo que aprendem muito mais com as outras oficinas [talleres] de: Português, Matemática, Rádio, Judô e o Programa Saúde na Escola (PSE). (Comunicação em Outras Palavras)

\subsection{Representación de los participantes}

Por esa alineación al discurso escolar o al discurso del periodismo hegemónico, los roles desempeñados también se ajustan a esos perfiles. De este modo, los roles típicos de los periódicos argentinos aquí estudiados son el/la estudiante y el/la profesor/a, como se verifica en este fragmento:

(19) En el marco de nuestro proyecto de Ciencias Naturales tenemos pensado actuar para ayudar a la ecología. Los temas que más nos preocupan son la tala de árboles y el problema de la basura. (Somos Argentinos: 12)

El enunciador se presenta como alumno y como alguien que se preocupa por un tema escolar (la ecología y la defensa del planeta), se coloca además como alguien que reflexiona sobre el mundo ("pensado actuar"), que elige y jerarquiza temas ("temas que más nos preocupa"). Al mismo tiempo, ese modo de enunciarse lo muestra junto a la voz de los/las docentes ("nuestro proyecto").

En los periódicos brasileños estudiados, el enunciador se presenta como periodista niño. Es un rol que comprende la representación del enunciador como mediador comunicacional y por tanto la función de relatar los eventos, tomándolos como existentes por sí mismos ("virou" - quedo-se), sin una voz más personal o reflexiva.

(20) No dia 14 de julho a Escola Vitor Manuel virou um grande "arraiá" [un pueblo compesino]. Alunos, professores e funcionários confeccionaram a decoração e se caracterizaram para a grande festa. (Comunicação em Outras Palavras: 2)

El enunciador también se posiciona como animador de un auditorio; de ahí que, se supone, los eventos deban ser relatados como grandes ("grande 'arraiá'”, "grande festa"). 


\section{Consideraciones finales}

La representación hegemónica del periodismo que viene de los medios de comunicación dominantes es muy poderosa y se disfraza con otros colores que la hacen imperceptible para vastos sectores sociales cuando se trata de leerla críticamente. En términos del presente análisis, se observa que, en las políticas de enseñanza de prácticas periodísticas, tanto en Argentina como en Brasil, son adoptados los dogmas comunicacionales dominantes, principalmente las suposiciones de neutralidad e imparcialidad de la prensa. Los dogmas se desarrollan mediante un discurso técnico que elimina la agentividad política, transformando la comunicación masiva en un lenguaje supuestamente guiado por leyes universales, que garantizarían la existencia de los regímenes democráticos. De este modo la representación dominante de la práctica se introduce en las escuelas como una formación para la ciudadanía, término que también sostiene un sentido técnico, y no político.

La representación crítica de los medios, aunque aparezca en los documentos curriculares de los dos municipios enfocados, es poco potente y necesita todavía ganar más cuerpo como política y como conjunto de acciones en las escuelas.

En términos de los periódicos estudiados (los argentinos, que tienden más al discurso escolar, y los brasileños, que tienden más al discurso de los medios dominantes), es importante señalar el valor y la necesidad de proseguir con esas actividades aunque despierten debates e críticas. Las críticas solamente son posibles porque algunos/as corajosamente empezaron a hacer ese trabajo, y el debate es necesario para desarrollarlo. Sería interesante que la producción de periódicos escolares comprendiera cada vez más un trabajo de análisis social de los medios tanto de los textos periodísticos cuanto del papel social e económico de las empresas de comunicación.

Como se desarrollan actualmente, los Programas de Enseñanza de Prácticas Periodísticas funcionan como culto y como catecismo de los dogmas de las empresas de comunicación. La reflexión sobre el periodismo, sin embargo, es importantísima, considerando el poder que los medios dominantes tienen para imponer sus principios y el razonamiento de determinado grupo sobre los demás. La lectura crítica de los medios es la clave, y para que ella ocurra es necesario que los/las estudiantes tengan la oportunidad de percibir la diferencia entre proyectos empresariales, colectivos y estatales de gestión de la comunicación social. Es importante, por lo tanto, que las políticas de enseñanza así como los medios producidos evidencien ese debate. 


\section{Q Bibliografía}

》Albarello, Francisco. 2011. El periódico escolar como proyecto institucional colaborativo. Fecha de consulta, 12 de octubre de 2017.

》http://newsmatic.com.ar/conectar/archivos/10/pe1a1_clase2_2_peri_dico.pdf.

》 Alvarado, M. (Coord.) 2001. Entre líneas. Teorías y enfoques de la enseñanza de la escritura, la gramática y la literatura. Buenos Aires: Manantial.

" Argentina. Presidencia de la Nación. Ministerio de Educación. Núcleos de Aprendizajes Prioritarios: Séptimo año - $7^{\circ}$ año educación primaria/ $1^{\circ}$ año educación secundaria. Buenos Aires: Ministerio de Educación 2011.

》 Argentina. 2006a. Congreso Nacional. Ley de Educación Nacional 26.206.

» Argentina. 2006b. Presidencia de la Nación. Ministerio de Educación Ciencia y Tecnología. Los medios de comunicación en la escuela: Un abordaje reflexivo, una actitud crítica. Buenos Aires: Ministerio de Educación.

» Argentina. 2005. Presidencia de la Nación. Ministerio de Educación Ciencia y Tecnología. Núcleos de Aprendizajes Prioritarios: $2^{\circ}$ ciclo EGB/ nivel primario - $4^{\circ}, 5^{\circ}$ y $6^{\circ}$ años. Buenos Aires: Ministerio de Educación Ciencia y Tecnología.

» Argentina. 1995. Presidencia de la Nación. Ministerio de Cultura y Educación de la Nación. Contenidos básicos comunes para la educación general básica. 2. ed. Buenos Aires: Ministerio de Cultura y Educación de la Nación.

"Arnoux, Elvira Narvaja de. 2015a. "Glotopolítica: Delimitación del campo y discusiones actuales con particular referencia a Sudamèrica." En Lengua y política en América Latina: Perspectivas actuales; Actas del II Coloquio Internacional de Estudios Latinoamericanos de Olomouc (CIELO2), editado por Lenka Zajícová y Radim Zámec. Olomouc: Univerzita Palackého v Olomouci, en prensa.

》Arnoux, Elvira N. de. 2015b. "Hacia una política de la lectura desde la perspectiva de la integración regional. Enfoque glotopolítico.” En Literatura-Lingüística Investigaciones en la Patagonia VIII, comp. por Marta Sotes, en soporte CD, 450-465. Comodoro Rivadavia: EDUPA - Trelew: ILLPAT.

" Arnoux, Elvira Narvaja de. 2015c. "Los manuales de estilo periodísticos para las versiones on line: Las representaciones del lector y su incidencia en la regulación de discursos y prácticas." Circula: Revue d'idéologies linguistiques 2: 138-160.

"Arnoux, Elvira N. de y José del Valle. 2010. "Las representaciones ideológicas del lenguaje. Discurso glotopolítico y panhispanismo." Spanish in Context número especial sobre "Ideologías lingüísticas: 1-24.

" Bajtín, Mijaíl. [1953] 1985. "El problema de los géneros discursivos." En Estética de la creación verbal, 248-293. México: Siglo XXI editores.

" Bonini, Adair. 2017. "O jornal escolar como mídia contra-hegemônica: Jornalismo de escola não modelado pelo jornalismo comercial dominante." Linguagem em (Dis)curso 17, n. 2: 165-182.

" Bonini, Adair. 2013. "Análise crítica de gêneros discursivos no contexto das práticas jornalísticas.” En Gêneros: Um diálogo entre comunicação e Linguística Aplicada, organizado por Lia Seixas y Najara Ferrari Pinheiro, 103-120. Florianópolis: Insular.

"Bonini, Adair. 2010. Critical genre analysis and professional practice: The case of public 
contests to select professors for Brazilian public universities. Linguagem em (Dis)curso 10, n. 3: 485-510.

" Bonini, Adair. 2008. "As relações constitutivas entre o jornal e seus gêneros: relato das pesquisas do Projeto Gêneros do Jornal". En Ciências da linguagem: avaliando o percurso, abrindo caminhos, organizado por Sandro Braga et. al., 21-45. Blumenau: Nova Letra.

" Brasil. 2017. Ministério da Educação. Secretaria de Educação Básica. Base nacional comum curricular: Educação infantil e ensino fundamental. Brasília: MEC/SEB.

» Brasil. 2013. Ministério da Educação. Secretaria de Educação Básica. Manual operacional de educação integral. Brasília: MEC/SEB.

" Brasil. 2011. Ministério da Educação. Secretaria de Educação Básica. Guia de Tecnologias Educacionais 2011/2012. Brasília: MEC/SEB.

» Brasil. 1998. Ministério da Educação. Secretaria de Educação Fundamental. Parâmetros curriculares nacionais: Terceiro e quarto ciclos do ensino fundamental: Língua Portuguesa. Brasília: MEC/SEF.

》 Britto, Luiz Percival Leme. 1997. A sombra do caos. Campinas/SP: Mercado das Letras.

"Buckingham, David. 2003. Media education: Literacy, learning and contemporary culture. Cambridge, UK: Polity Press - Oxford, UK: Blackwell.

"Buenos Aires. 2004. Gobierno de la Ciudad Autónoma de Buenos Aires. Secretaria de Educación. Diseño curricular para la escuela primaria: Segundo ciclo de la escuela primaria/ educación general básica, publicada en 2 tomos. Buenos Aires: GCBA Secretaria de Educación.

"Buenos Aires. s.f. Medios en la escuela. Fecha de consulta, 12 de octubre de 2017.

"www.buenosaires.gob.ar/areas/educacion/niveles/primaria/programas/medios_en_la_ escuela.

" Comunicação e Cultura. 2010. Guia do jornal escolar. Elaboración, texto e edición de Daniel Raviolo. Fortaleza: Comunicação e Cultura.

" Coscarelli, Carla Viana y Ana Elisa Ribeiro. Letramento Digital - Aspectos Sociais e Possibilidades Pedagógicas. Belo Horizonte: Autêntica Editora, 2007.

》 Cosson, R. Letramento literário: Teoria e prática. São Paulo: Contexto, 2006.

" Fairclough, N. Analyzing discourse: Textual analysis for social research. London: Routledge, 2003.

" Ferretti-Soares, Vanessa Arlésia de Souza y Adair Bonini. 2017. "A representação estratégica da violência urbana na mídia: Uma análise crítica de gênero." Cadernos de Linguagem e Sociedade 18, n. 3: 313-332.

» Florianópolis. 2016. Prefeitura Municipal de Florianópolis. Secretaria de Educação. Proposta Curricular da Rede Municipal de Ensino de Florianópolis - 2016, organizado por Claudia Cristina Zanela, Ana Regina Ferreira de Barcelos e Rosângela Machado. Florianópolis: PMF.

"Florianópolis. 2008. Prefeitura Municipal de Florianópolis. Secretaria Municipal de Educação. Departamento de Educação Fundamental. Proposta Curricular. Florianópolis: PMF.

》Freire, Paulo. 1967. Educação como prática de liberdade. Rio de Janeiro: Paz e Terra.

" Geraldi, João Wanderley. 1991. Portos de passagem, 4. ed. São Paulo: Martins Fontes.

» HALTÉ, Jean-François. [1998] 2008. "O espaço didático e a transposição." En Fórum Lingüístico 5, n. 2: 117-139. 
Kaufman, Ana María y María Elena Rodríguez. 1993. La escuela y los textos. Buenos Aires: Santillana.

" Marcellesi, Jean-Baptiste y Louis Guespin. 1986. “Pour la Glottopolitique.” Langages 83: 5-34.

» Morduchowicz, Roxana. 2009. Cuando la educación en medios es política de estado. Comunicar XVI, n. 32: 131-138.

" Morduchowicz, Roxana. 2001. El diario en la escuela. Barcelona: Octaedro.

» Morduchowicz, Roxana, Atilio Marcón y Viviana Minzl. 2004. La escuela y los medios: Propuestas para explorar los medios en la escuela. Buenos Aires: Ministerio de Educación, Ciencia y Tecnología.

" Motta-Roth, Désirée y Viviane Maria Heberle. 2015. "A short cartography of genre studies in Brazil." Journal of English for Academic Purposes 19: 22-31.

» Rechetnicou, Amanda, Sostenes Lima y Adair Bonini. 2016. "Blog jornalístico e a produção do discurso de resistência nas práticas de leitura e escrita." Revelli: Revista de Educação, Linguagem e Literatura da UEG-Inhumas 8: 145-165.

"Street, B. 1984. Literacy in theory and practice. Cambridge: Cambridge University Press.

» Unesco. 2011. Alfabetización mediática e informacional: Curriculum para profesores. París: UNESCO.

»Voese, Ingo. 2002. "Desafios para uma análise do discurso (e para o ensino?)." Linguagem em (Dis)curso 3, n. 1: 187-210. 